\title{
Studies with enamines: Functionally substituted enamines as aldehyde equivalents in Gewald reactions
}

\author{
Saleh Al-Mousawi, ${ }^{*}$ Moustafa Sherief Moustafa, and Mohamed Hilmy Elnagdi \\ Department of Chemistry, Faculty of Science, University of Kuwait: P.O. Box 5969: Safat: \\ 13060, Kuwait \\ E-mail: salehalmousawi@hotmail.com
}

\begin{abstract}
Several aryl and functionally substituted enamines reacted with ethyl cyanoacetate and elemental sulfur to form 2-aminothiophene-3-carboxylic acid derivatives that proved to be excellent precursors for a variety of thiophenes.
\end{abstract}

Keywords: Gewald reaction, enamines, elemental sulfur, thiophenes

\section{Introduction}

In 1966 Gewald $^{1}$ reported that aliphatic ketones, aldehydes or 1,3-dicarbonyl compounds react with activated nitriles and sulfur in the presence of a base at room temperature to give 2aminothiophenes. ${ }^{1-4}$ Since aminothiophenes are important intermediates in dye preparations, ${ }^{5,6}$ in the pharmaceutical industry and as a precursors for other thiophenes employed in several high technology applications, ${ }^{7}$ this methodology has been extensively utilized for the preparation of a variety of aminothiophenes via reacting ketones and aldehydes with active methylene carbonitriles and sulfur in presence of a base. ${ }^{8-11}$ In the context of our interest in aminothiophenes as precursors to arylazo dyes ${ }^{12,13}$ we became interested in 2-amino-4unsubstituted thiophenes with a functional substituent at C-5.

\section{Results and Discussion}

The logical starting materials for such thiophenes would be $\beta$-functional aldehydes $\mathbf{1}$, active methylene nitriles 2 and elemental sulfur (cf. equation 1). However as $\beta$-functionalized aldehydes are rather unstable compounds therefore, we considered the possible use of functionally substituted enamines as their synthetic equivalents. We have, in the last decade, utilized such functionally substituted enamines extensively as precursors to functionally 
substituted aromatic compounds ${ }^{14,15}$ and heteroaromatic compounds. ${ }^{16-20}$ We noted that only a few examples of the use of enamines $\mathbf{3} \mathbf{a}$ as precursors to aminothiophenes have been reported. ${ }^{9,21}$

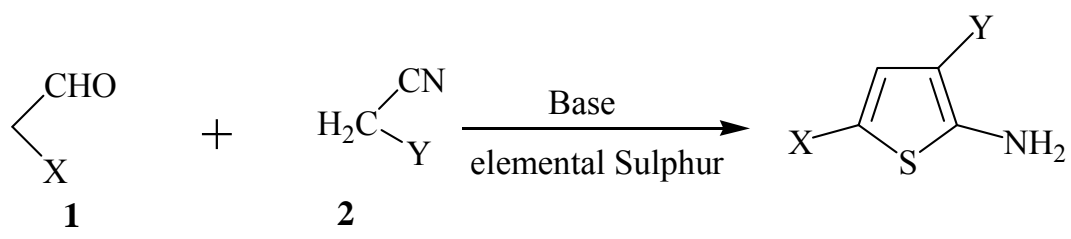

\section{Equation 1}

We envisioned that the initial step in this sequence would be the addition of an active methylenenitrile to the $\alpha, \beta$-unsubstituted moiety in $\mathbf{3}$ with subsequent elimination of diethylamine to yield 4A or 4B. Alternatively, reaction of adduct 7 with sulfur in the presence of an equivalent amount of piperidine would yield $\mathbf{8}$ that would cyclise and aromatize to yield $\mathbf{6}$. This sequence is quite similar to the reported general mechanism of the Gewald reaction. ${ }^{9}$ In support of this we noted the elimination of $\mathrm{H}_{2} \mathrm{~S}$ during the reaction. In either case, the activity of methylene moiety in either 4 or 7 is essential for the success of reaction. Consequently we selected enamines 3b-f with electron attracting substituents to fulfill this prerequisite. We found that, 3b-e reacted smoothly with elemental sulfur and ethyl cyanoacetate in the presence of equivalent amounts of piperidine (cf. Experimental Section) to yield amino thiophenes $\mathbf{6 b}-\mathbf{e}, \mathbf{6 b}$ in $70 \%, 6 \mathbf{c}, 73 \%, \mathbf{6 d}, 76 \%$ and $\mathbf{6 e}, 79 \%$ yields. Treating $3 \mathbf{f}$ similarly with sulfur and ethyl cyanoacetate resulted in the formation of 2-aminothiophene-5-thiocarboxamide $6 \mathrm{~g}$ in $60 \%$ yield. We believe that initially $\mathbf{6 f}$ is formed and this reacted further with the hydrogen sulfide produced during the reaction to yield the thioamide $\mathbf{6 g}$ (cf. Scheme 1). Attempts to replace ethyl cyanoacetate by malononitrile failed in our hands to yield pure isolable products. An oily mixture of several products was produced with this reactant. Structures 6, taking 6c as a typical example, are supported by spectral and analytical evidence. Thus the MS of the typical example showed an $\mathrm{M}^{+}$peak at $309(100)$, two intess peaks at $263(100)\left(\mathrm{M}^{+}-\mathrm{OC}_{2} \mathrm{H}_{5}\right)$, and $139(100)(\mathrm{P}-$ chlorobenzoyl cation). Its ${ }^{13} \mathrm{C}$ NMR spectrum showed two signal for $\mathrm{sp}^{3}$ carbons of the ester group at $\delta=15.05 \mathrm{ppm}$ and $\delta=60.99 \mathrm{ppm}$; a benzyl carbonyl carbon at $\delta=186.61 \mathrm{ppm}$, ester carbonyl carbon and C-2 at $\delta=169.32 \mathrm{ppm}$ and $\delta=165.64 \mathrm{ppm}$ (not necessarily respectively) and seven carbon at $\delta=138.56,137.37,136.81,130.68,129.35,125.26,109.21 \mathrm{ppm}$. The ${ }^{1} \mathrm{H}$ NMR spectrum also showed the expected signals for ethyl ester as triplets and quartets at $\delta=$ 1.35 and $\delta=4.31 \mathrm{ppm}$ respectively. The thiophene $\mathrm{C}-4$ proton signal appeared as a singlet at $\delta=$ $7.57 \mathrm{ppm}$ and an amino, $\mathrm{D}_{2} \mathrm{O}$ exchangeable, signal at $\delta=6.74 \mathrm{ppm}$. The other analogues had spectra in complete agreement with proposed structure. The analytical data fits perfectly the proposed structures. 


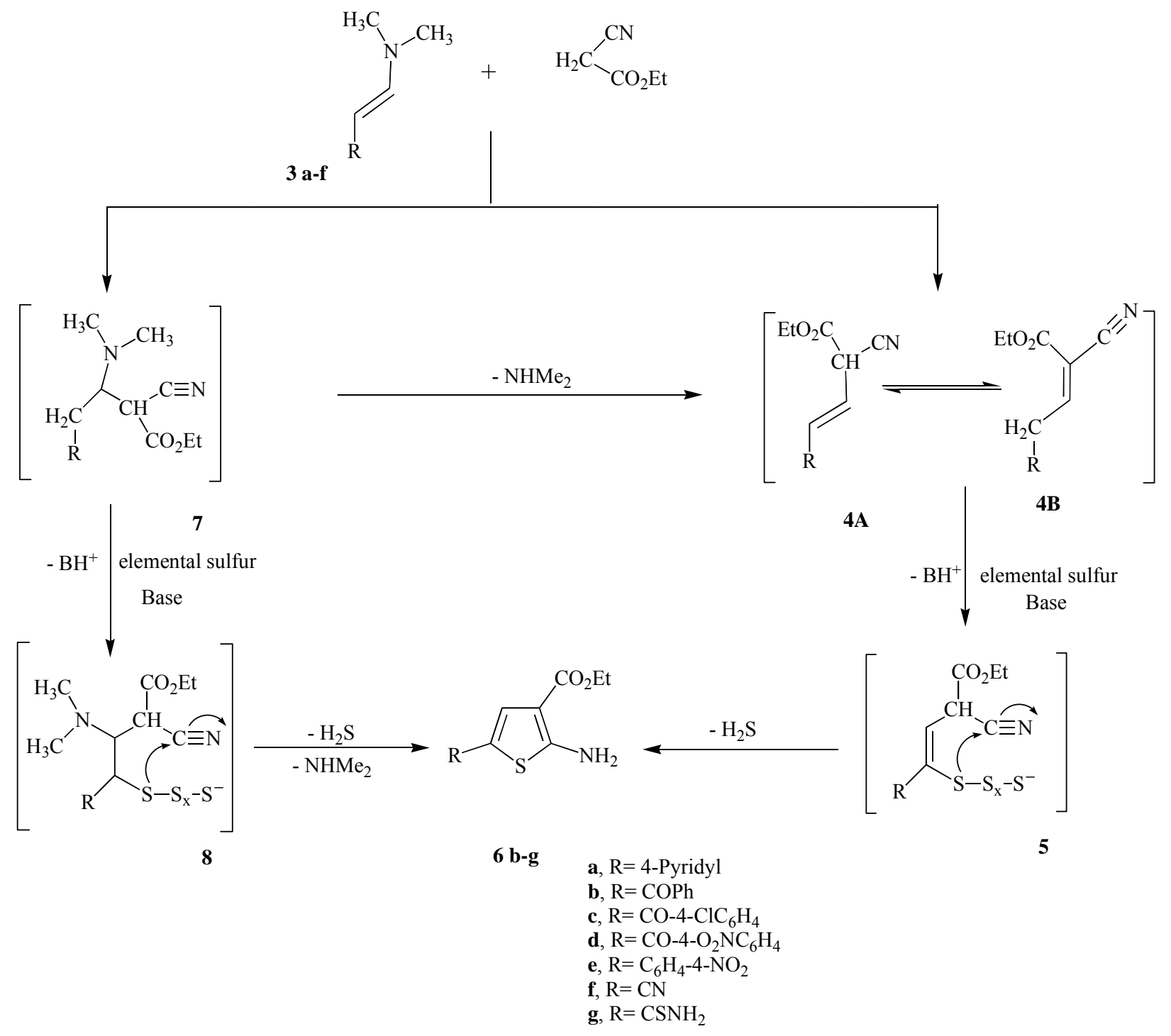

\section{Scheme 1}

The 2-aminothiophene-5-carboxylic acid esters $\mathbf{6 b}$-g proved to be excellent precursors for the synthesis of arylazothiophenes as well as thieno[2,3-d]pyrimidines. Thus diazotizing 6b,e in the presence of hydrochloric acid in acetic acid solution afforded diazonium salts that readily coupled with malononitrile to yield a thienylhydrazonomesoxalonitriles 9b,e. Reacting 9b with hydrazine hydrate gave the 3,5-diaminopyrazoles 10b. Arylazopyrazol-3,5-diamines prepared long ago $^{22}$ in our laboratories have recently been shown to have interesting antiprofilerative ${ }^{23}$ activity and were also patented for possible utility as oxidative dyes for keratin fiber and for hair. ${ }^{24}$ Thienylpyrazole-3,5-diamines merit testing in both these areas and this will be carried out. Aminothiophenes $\mathbf{6 b}$ also condensed with dimethylformamide dimethylacetal to yield amidines 11b that reacted in turn with acetic acid and ammonium acetate to yield a 1:1 mixture of 
thienopyrimidine 12b and acetylaminothiophene 13b. The latter could also be obtained via acylation of $\mathbf{6 b}$ with acetic anhydride (cf. Scheme 2).

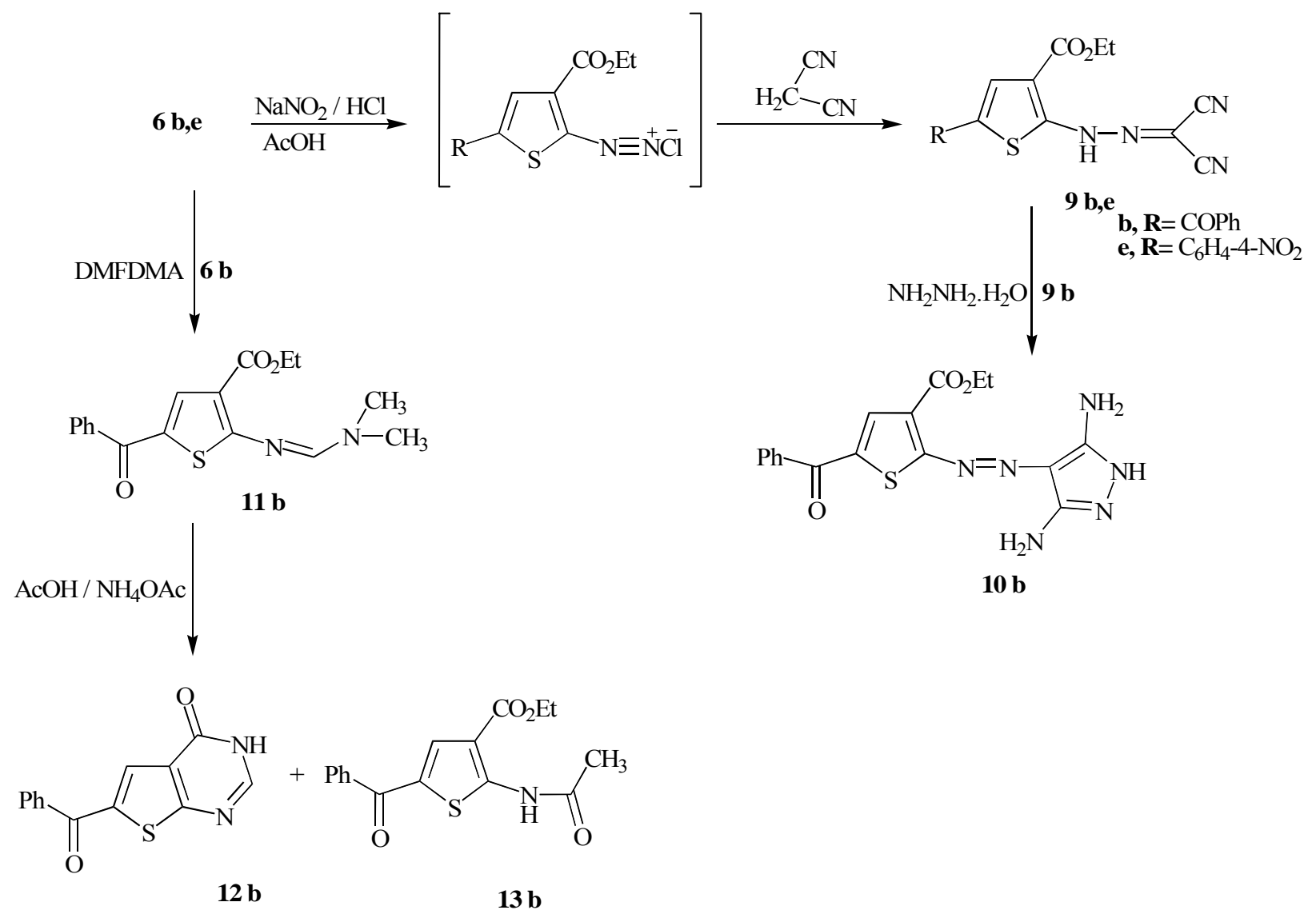

\section{Scheme 2}

\section{Conclusions}

In conclusion we have shown that functionally substituted enamines are excellent precursors for the synthesis of 2-amino-5-functionally-substituted thiophene carboxylic acid esters that can be readily used as precursors to potentially interesting thiophenes.

\section{Experimental Section}

General Procedures. All melting points are uncorrected and were determined with a Sanyo (Gallaenkamp) instrument. Infrared spectra were recorded in $\mathrm{KBr}$ and were determined on a Perkin-Elmer 2000 FT-IR system. ${ }^{1} \mathrm{H}$ NMR and ${ }^{13} \mathrm{C}$ NMR spectra were determined on a Bruker 
DPX at (400 MHz for ${ }^{1} \mathrm{H}$ NMR and $100 \mathrm{MHz}$ for ${ }^{13} \mathrm{C} \mathrm{NMR}$ ) spectrometer in $\mathrm{CDCl}_{3}$ or DMSO-d as solvent and TMS as internal standard; chemical shifts are reported in $\delta$ (ppm). Mass spectra were measured on VG Autospec Q MS 30 and MS 9 (AEI) spectrometers, with EI 70 EV. Elemental analyses were measured by means of LEOCHNS-932 Elemental Analyzer.

\section{General procedure to synthesis compounds 6b-e}

A mixture of $3 \mathbf{b}(1.75 \mathrm{~g}, 0.010 \mathrm{~mol})$, ethyl cyanoacetate $(1.13 \mathrm{~g}, 0.010 \mathrm{~mol})$ and elemental sulfur $(0.32 \mathrm{~g}, 0.010 \mathrm{~mol})$ in dry $N, N$-dimethylformamide as solvent $(10 \mathrm{~mL})$ was treated with an of equivalent amounts of piperidine $(2 \mathrm{~mL})$. The reaction mixture was refluxed for $6-8 \mathrm{~h}$, cooled and then poured onto ice-water. The residue, so formed, was extracted by dichloromethane. The organic layer was dried and the solvent was evaporated. The residue was purified through coloumn chromatography on silica gel using a mixture of petroleum ether (60-80) and ethyl acetate $(3: 1)$ as an eluent.

Ethyl 2-amino-5-benzoylthiophene-3-carboxylate (6b). Formed as yellow crystals in 70\% yield; mp. 141-42 ${ }^{\circ} \mathrm{C}$. Anal. Calcd. for $\mathrm{C}_{14} \mathrm{H}_{13} \mathrm{NO}_{3} \mathrm{~S}$ (275.06): C, 61.0; H, 4.7; N, 5.0; S, 11.7\% Found: C, 60.8; H, 4.7; N, 5.1; S, 11.9\% IR (KBr, cm $\left.{ }^{-1}\right): 3298,3262\left(\mathrm{NH}_{2}\right), 1686(\mathrm{CO}), 1620$ $(\mathrm{CO}) ;{ }^{1} \mathrm{H} \mathrm{NMR}\left(400 \mathrm{MHz}, \mathrm{CDCl}_{3}\right): \delta, \mathrm{ppm}=1.28\left(\mathrm{t}, 3 \mathrm{H}, \mathrm{CH}_{3}, J=8 \mathrm{~Hz}\right), 4.31$ (q, $2 \mathrm{H}, \mathrm{CH}_{2}, J=8$ $\mathrm{Hz}), 6.60$ (br, 2H, $\mathrm{NH}_{2}$ ) $\mathrm{D}_{2} \mathrm{O}$ exchangeable, 7.49-7.81 (m, 6H, Ar-H, CH-thiophene); ${ }^{13} \mathrm{C}$ NMR (100 MHz, DMSO-d6): $\delta$, ppm = $15.1\left(\mathrm{CH}_{3}\right), 60.5\left(\mathrm{CH}_{2}\right), 107.3,123.5,128.9,129.3,164.7$, 170.2 (CO), 186.8 (CO). MS: m/z (\%) $275\left(\mathrm{M}^{+}, 100\right), 229$ (95), 198 (20), 152 (40), 105 (80), 77 (60).

Ethyl 2-amino-5-(4-chlorobenzoyl)thiophene-3-carboxylate (6c). Formed as yellow crystals in $73 \%$ yield; mp. $151-52{ }^{\circ} \mathrm{C}$. Anal. Calcd. for $\mathrm{C}_{14} \mathrm{H}_{12} \mathrm{ClNO}_{3} \mathrm{~S}$ (309.77): C, 54.2; H, 3.9; N, 4.5; S, 10.3\% Found: C, 54.3; H, 3.9; N, 4.8; S, 10.3\% IR (KBr, cm $\left.{ }^{-1}\right): 3396,3297\left(\mathrm{NH}_{2}\right), 1677$ (CO), 1600 (CO); ${ }^{1} \mathrm{H}$ NMR (400 MHz, $\left.\mathrm{CDCl}_{3}\right): \delta, \mathrm{ppm}=1.35$ (t, 3H, $\left.\mathrm{CH}_{3}, J=8 \mathrm{~Hz}\right), 4.31$ (q, 2H, $\mathrm{CH}_{2}$, $J=8 \mathrm{~Hz}$ ), 6.74 (br, 2H, $\left.\mathrm{NH}_{2}\right) \mathrm{D}_{2} \mathrm{O}$ exchangeable, 7.49 (d, 2H, Ar-H, $\left.J=8 \mathrm{~Hz}\right), 7.57$ (s, 1H, CHthiophene ), $7.74(\mathrm{~d}, 2 \mathrm{H}, \mathrm{Ar}-\mathrm{H}, J=8 \mathrm{~Hz}) ;{ }^{13} \mathrm{C}$ NMR (100 MHz, DMSO-d6): $\delta, \mathrm{ppm}=15.0$ $\left(\mathrm{CH}_{3}\right), 60.9\left(\mathrm{CH}_{2}\right), 109.2,125.2,129.3,130.6,136.8,137.3,138,5,165.6,169.3(\mathrm{CO}), 186.6$ (CO). MS: m/z (\%) 309 (M+1 100), 263 (100), 228 (20), 198 (20), 152 (55), 139 (100), 111 (80), 97 (15), 75 (30).

Ethyl 2-amino-5-(4-nitrobenzoyl)thiophene-3-carboxylate (6d). Formed as yellow crystals in $76 \%$ yield; mp. $205-07{ }^{\circ} \mathrm{C}$. Anal. Calcd. for $\mathrm{C}_{14} \mathrm{H}_{12} \mathrm{~N}_{2} \mathrm{O}_{5} \mathrm{~S}$ (320.32): C, 52.5; H, 3.7; N, 8.7; S, 10.0\% Found: C, 52.6; H, 3.9; N, 8.7; S, 10.0\% IR (KBr, cm $\left.{ }^{-1}\right): 3401,3273\left(\mathrm{NH}_{2}\right), 1689$ (CO), 1623 (CO); ${ }^{1} \mathrm{H}$ NMR (400 MHz, $\left.\mathrm{CDCl}_{3}\right): \delta, \mathrm{ppm}=1.33$ (t, 3H, $\left.\mathrm{CH}_{3}, J=8 \mathrm{~Hz}\right), 4.31$ (q, 2H, $\mathrm{CH}_{2}$, $J=8 \mathrm{~Hz}), 6.79\left(\mathrm{br}, 2 \mathrm{H}, \mathrm{NH}_{2}\right) \mathrm{D}_{2} \mathrm{O}$ exchangeable, 7.54 (s, 1H, CH-thiophene ), 7.93 (d, 2H, Ar$\mathrm{H}, J=8 \mathrm{~Hz}), 8.37$ (d, 2H, Ar-H, $J=8 \mathrm{~Hz}) ;{ }^{13} \mathrm{C}$ NMR (100 MHz, DMSO-d6): $\delta$, ppm = 14.5 $\left(\mathrm{CH}_{3}\right), 60.6\left(\mathrm{CH}_{2}\right), 109.1,123.8,124.2,129.5,137.6,143.4,149.5,164.9,169.3(\mathrm{CO}), 185.1$ (CO). MS: $m / z(\%) 320\left(\mathrm{M}^{+}, 100\right), 274$ (70), 229 (20), 152 (30), 104 (20), 96 (15), 76 (15). 
Ethyl 2-amino-5-(4-nitrophenyl)thiophene-3-carboxylate (6e). The solid product, so formed, was crystallized from ethanol to yield a brown product, $79 \%$ yield; mp. $164-65{ }^{\circ} \mathrm{C}$. Anal. Calcd. for $\mathrm{C}_{13} \mathrm{H}_{12} \mathrm{~N}_{2} \mathrm{O}_{4} \mathrm{~S}$ (292.05): C, 53.4; H, 4.1; N, 9.5; S, 10.9\% Found: C, 53.2; H, 4.2; N, 9.7; S, $10.8 \%$ IR (KBr, cm $\left.{ }^{-1}\right): 3443,3232\left(\mathrm{NH}_{2}\right), 1671(\mathrm{CO}) ;{ }^{1} \mathrm{H}$ NMR $\left(400 \mathrm{MHz}, \mathrm{DMSO}-\mathrm{d}_{6}\right): \delta, \mathrm{ppm}=$ 1.29 (t, 3H, $\mathrm{CH}_{3}, J=8 \mathrm{~Hz}$ ), 4.29 (q, 2H, $\mathrm{CH}_{2}, J=8 \mathrm{~Hz}$ ), 7.59 (s, 1H, CH-thiophene), 7.68 (d, $2 \mathrm{H}, \mathrm{Ar}-\mathrm{H}, J=8 \mathrm{~Hz}$ ), $7.82\left(\mathrm{br}, 2 \mathrm{H}, \mathrm{NH}_{2}\right) \mathrm{D}_{2} \mathrm{O}$ exchangeable, $8.14(\mathrm{~d}, 2 \mathrm{H}, \mathrm{Ar}-\mathrm{H}, J=8 \mathrm{~Hz}) ;{ }^{13} \mathrm{C}$ NMR (100 MHz, DMSO-d6): $\delta, \mathrm{ppm}=15.31,60.25,106.76,120.25,124.92,125.36,126.82$, 141.31, 145.33, 164.91, 166.06. MS: m/z (\%) $292\left(\mathrm{M}^{+}, 85\right), 246$ (95), 215 (25), 171 (20), 144 (15).

Synthesis of ethyl 2-amino-5-carbamothioylthiophene-3-carboxylate (6g). A mixture of 3d $(1.36 \mathrm{~g}, 0.010 \mathrm{~mol})$, ethyl cyanoacetate $(1.13 \mathrm{~g}, 0.010 \mathrm{~mol})$ and elemental sulfur $(0.32 \mathrm{~g}, 0.010$ mol) in N,N-dimethylformamide as solvent $(10 \mathrm{~mL})$ was treated with a few drops of piperidine. The reaction mixture was refluxed for 6-8 h., cooled and then poured onto ice-water. The residue, so formed, was extracted by dichloromethane. After evaporation of dichloromethane the residue was purified by column chromatography using a mixture of petroleum ether (60-80) and ethyl acetate $(5: 1)$ as an eluent. The afforded solid product was crystallized from diethyl ether to give yellow product; yield $60 \%$; mp 214-15 ${ }^{\circ} \mathrm{C}$. Anal. Calcd. for $\mathrm{C}_{8} \mathrm{H}_{10} \mathrm{~N}_{2} \mathrm{O}_{2} \mathrm{~S}_{2}$ (230.01): C, 42.3; H, 4.3; N, 12.1; S, 27.8\% Found: C, 42.3; H, 4.5; N, 12.3; S, 27.7\% IR (KBr, $\left.\mathrm{cm}^{-1}\right): 3376$, $3193\left(\mathrm{NH}_{2}\right), 3273,3153\left(\mathrm{NH}_{2}\right), 1679(\mathrm{CO}) ;{ }^{1} \mathrm{H} \mathrm{NMR}\left(400 \mathrm{MHz}, \mathrm{DMSO}-\mathrm{d}_{6}\right): \delta, \mathrm{ppm}=1.26(\mathrm{t}$, $3 \mathrm{H}, \mathrm{CH}_{3}, J=8 \mathrm{~Hz}$ ), 4.20 (q, 2H, $\mathrm{CH}_{2}, J=8 \mathrm{~Hz}$ ), 7.68 (s, 1H, CH-thiophene), 7.85 (br, 2H, $\mathrm{NH}_{2}$ ) $\mathrm{D}_{2} \mathrm{O}$ exchangeable, $9.08\left(\mathrm{br}, 2 \mathrm{H}, \mathrm{NH}_{2}\right) \mathrm{D}_{2} \mathrm{O}$ exchangeable; ${ }^{13} \mathrm{C}$ NMR (100 MHz, DMSO-d 6 ): $\delta$, $\mathrm{ppm}=15.4,60.3,106.9,126.5,165.5,170.3,170.5,189.3 . \mathrm{MS}: m / z(\%) 230\left(\mathrm{M}^{+}, 100\right), 197$ (35), 184 (90), $168(20), 151(60), 123$ (15), 96 (30), 69 (20).

\section{General procedures for preparation of compounds $9 b, e$}

A solution of $\mathbf{6 b}$ or $\mathbf{6 e}(0.010 \mathrm{~mol})$ in acetic acid $(8 \mathrm{~mL})$, was treated with concentrated hydrochloric acid $(3 \mathrm{~mL})$ and sodium nitrite $(0.69 \mathrm{~g}, 0.010 \mathrm{~mol})$ at $0{ }^{\circ} \mathrm{C}$, This mixture was added gradually with stirring, to a cooled solution of malononitrile $(0.66 \mathrm{~g}, 0.010 \mathrm{~mol})$ in ethanol $(10$ $\mathrm{mL})$ and sodium acetate $(1.0 \mathrm{~g})$. After complete addition, the reaction mixture was kept at room temperature for one hour. The solid product, so formed, was collected by filtration.

5-Benzoyl-2-( $N^{\prime}$-dicyanomethylene-hydrazino)-thiophene-3-carboxylic acid (9b). The collected solid product was crystallized from ethanol to yield yellow product, $86 \%$ yield; mp. 181-82 ${ }^{\circ} \mathrm{C}$. Anal. Calcd. for $\mathrm{C}_{17} \mathrm{H}_{12} \mathrm{~N}_{4} \mathrm{O}_{3} \mathrm{~S}$ (352.06): C, 57.9; H, 3.4; N, 15.9; S, 9.1\% Found: C, 57.7; H, 3.6; N, 15.9; S, 8.9\% IR (KBr, cm $\left.{ }^{-1}\right): 3437$ (NH), 2235 (CN), 2212 (CN), 1679 (CO), $1631(\mathrm{CO}) ;{ }^{1} \mathrm{H}$ NMR (400 MHz, DMSO-d 6 ): $\delta, \mathrm{ppm}=1.42\left(\mathrm{t}, 3 \mathrm{H}, \mathrm{CH}_{3}, J=8 \mathrm{~Hz}\right), 4.45$ (q, 2H, $\mathrm{CH}_{2}, J=8 \mathrm{~Hz}$ ), 7.55-7.87 (m, 6H, Ar-H, CH-thiophene), 12.7 (br, 1H, NH) $\mathrm{D}_{2} \mathrm{O}$ exchangeable; ${ }^{13} \mathrm{C} \mathrm{NMR}\left(100 \mathrm{MHz}, \mathrm{CDCl}_{3}\right): \delta, \mathrm{ppm}=14.7,62.8,93.3,107.6,111.3,115.0,129.2,129.4$, 133.2, 133.8, 136.2, 137.0, 158, 164.5, 187.6. MS: $m / z(\%) 352\left(\mathrm{M}^{+}, 60\right), 306(75), 275(20), 105$ (100), 77 (40). 
5-(4-Amino-phenyl)-2-( $N$ '-dicyanomethylene-hydrazino)-thiophene-3-carboxylic acid (9e). As in the previous (above) procedures the products was crystallized from ethyl acetate to yield a dark brown product, $86 \%$ yield; mp. $216-17{ }^{\circ} \mathrm{C}$. Anal. Calcd. for $\mathrm{C}_{16} \mathrm{H}_{11} \mathrm{~N}_{5} \mathrm{O}_{4} \mathrm{~S}$ (269.05): C, 52.0; H, 3.0; N, 18.9; S, 8.6\% Found: C, 52.1; H, 3.0; N, 18.8; S, 8.8\% IR (KBr, cm $\left.{ }^{-1}\right): 3299$ $(\mathrm{NH}), 2226(\mathrm{CN}), 2208(\mathrm{CN}), 1677(\mathrm{CO}) ;{ }^{1} \mathrm{H}$ NMR (400 MHz, DMSO-d 6 ): $\delta, \mathrm{ppm}=1.36(\mathrm{t}, 3 \mathrm{H}$, $\mathrm{CH}_{3}, J=8 \mathrm{~Hz}$ ), 4.36 (q, 2H, $\mathrm{CH}_{2}, J=8 \mathrm{~Hz}$ ), 7.85 (s, 1H, CH-thiophene), 7.94 (d, 2H, Ar-H), 8.22 (d, 2H, Ar-H), 10.9 (br, 1H, NH) $\mathrm{D}_{2} \mathrm{O}$ exchangeable; ${ }^{13} \mathrm{C}$ NMR (100 MHz, DMSO-d 6 ): $\delta$, $\mathrm{ppm}=14.16,60.77,133.8,123.14,124.4,125.5,126.0,129.5,139.6,145.8,148.6,163.66$, 168.2. MS: $m / z(\%) 269\left(\mathrm{M}^{+}, 60\right), 334$ (40), 292 (95), 246 (100), 215 (20), 172 (20), 120 (15), 72 (20).

Synthesis of 3-5-Diamino-4-[(5-benzoyl-3-ethoxycarbonyl-2-thienyl)azo]-1H-pyrazole (10b). Equimolar amounts of $\mathbf{9 b}(2.52 \mathrm{~g}, 0.010 \mathrm{~mol})$ and hydrazine hydrate $(0.5 \mathrm{~g}, 0.010 \mathrm{~mol})$ in ethanol $(10 \mathrm{~mL})$ were heated at reflux for $5 \mathrm{~h}$. After cooling, the reaction mixture was poured onto ice-water. The solid, so formed, was collected by filtration and crystallized from ethanol to give a red product; $85 \%$ yield; mp. $169-70{ }^{\circ} \mathrm{C}$. Anal. Calcd. for $\mathrm{C}_{17} \mathrm{H}_{16} \mathrm{~N}_{6} \mathrm{O}_{3} \mathrm{~S}$ (384.1): C, 53.1; H, 4.2; N, 21.8; S, 8.35\% Found: C, 53.0; H, 4.0; N, 21.9; S, 8.1\% IR (KBr, cm $\left.{ }^{-1}\right): 3422(\mathrm{NH})$, 3317, $3201\left(\mathrm{NH}_{2}\right), 3217,3159\left(\mathrm{NH}_{2}\right), 1702$ (CO), $1617(\mathrm{CO}) ;{ }^{1} \mathrm{H}$ NMR (400 MHz, $\left.\mathrm{CDCl}_{3}\right): \delta$, ppm $=1.31\left(\mathrm{t}, 3 \mathrm{H}, \mathrm{CH}_{3}, J=8 \mathrm{~Hz}\right), 4.26\left(\mathrm{q}, 2 \mathrm{H}, \mathrm{CH}_{2}, J=8 \mathrm{~Hz}\right), 4.28\left(\mathrm{br}, 2 \mathrm{H}, \mathrm{NH}_{2}\right) \mathrm{D}_{2} \mathrm{O}$ exchangeable, 4.29 (br, 2H, $\left.\mathrm{NH}_{2}\right) \mathrm{D}_{2} \mathrm{O}$ exchangeable, 7.28-7.85 (m, 6H, Ar-H, CH-thiophene), 11.28 (br, $1 \mathrm{H}, \mathrm{NH}) \mathrm{D}_{2} \mathrm{O}$ exchangeable; ${ }^{13} \mathrm{C}$ NMR $\left(100 \mathrm{MHz}, \mathrm{DMSO}-\mathrm{d}_{6}\right): \delta, \mathrm{ppm}=15.2,61.2$, 122.2, 123.4, 129.3 (2C), 129.4 (2C), 133.1, 133.6, 134.1, 137.2, 138.3, 162.2, 162.9, 173.4, 188.0. MS: $m / z(\%) 384\left(\mathrm{M}^{+}, 50\right), 368$ (10), 274 (65), 228 (70), 151 (20), 104 (100), 77 (80).

Synthesis of ethyl 5-benzoyl-2-[(dimethylamino)methyleneamino]thiophene-3-carboxylate (11b). A mixture of compound $6 \mathbf{b}(2.75 \mathrm{~g}, 0.010 \mathrm{~mol})$ and $N, N$-dimethylformamide dimethylacetal $(1.19 \mathrm{~g}, 0.010 \mathrm{~mol})$ in toluene $(20 \mathrm{~mL})$ was refluxed for $6 \mathrm{~h}$. The reaction mixture was evaporated under reduced pressure in vacuum yielding a crude product, which was crystallized from ethanol to give yellow crystals; yield $85 \%$; mp. $130-31{ }^{\circ} \mathrm{C}$. Anal. Calcd. for $\mathrm{C}_{17} \mathrm{H}_{18} \mathrm{~N}_{2} \mathrm{O}_{3} \mathrm{~S}$ (330.1): C, 61.8; H, 5.4; N, 8.4; S, 9.7\% Found: C, 62.0; H, 5.4; N, 8.6; S, 9.8\% IR $\left(\mathrm{KBr}, \mathrm{cm}^{-1}\right): 1711(\mathrm{CO}), 1622(\mathrm{CO}) ;{ }^{1} \mathrm{H}$ NMR (400 MHz, DMSO-d 6 ): $\delta, \mathrm{ppm}=1.22\left(\mathrm{t}, 3 \mathrm{H}, \mathrm{CH}_{3}\right.$, $J=8 \mathrm{~Hz}), 3.06\left(\mathrm{~s}, 3 \mathrm{H}, \mathrm{CH}_{3}\right), 3.16\left(\mathrm{~s}, 3 \mathrm{H}, \mathrm{CH}_{3}\right), 4.15\left(\mathrm{q}, 2 \mathrm{H}, \mathrm{CH}_{2}, J=8 \mathrm{~Hz}\right), 7.54-7.75(\mathrm{~m}, 6 \mathrm{H}$, Ar-H, CH-thiophene), 8.09 (s, 1H, CH); ${ }^{13} \mathrm{C}$ NMR (100 MHz, DMSO-d $\left.\mathrm{d}_{6}\right): \delta, \mathrm{ppm}=15.0,35.0$, $40.3,60.5,118.2,128.7,129.2,129.5,132.7,138.4,139.0,157.7,163.2,172.2,187.0 . \mathrm{MS}: \mathrm{m} / z$ (\%) $330\left(\mathrm{M}^{+}, 65\right), 285$ (20), 242 (20), 216 (10), 105 (95), 77 (100).

\section{General procedures for preparation of compounds $\mathbf{1 2 b}, \mathbf{1 3 b}$}

A mixture of compound 11b (3.30 g, $0.010 \mathrm{~mol})$, ammonium acetate $(2.30 \mathrm{~g}, 0.03 \mathrm{~mol})$ and acetic acid $(7 \mathrm{~mL})$ was refluxed for $4-6 \mathrm{~h}$ (monitored by TLC). The reaction mixture was cooled and poured onto ice-water. The solid product, so formed, was collected by filtration and washed with petroleum ether to extract the yellow product 12. The residue was crystallized from ethyl acetate to yield white product 11. 
6-Benzoylthieno[2,3-d]pyrimidin-4(3H)-one (12b). Yield 45\%, mp. 266-68 ${ }^{\circ} \mathrm{C}$. Anal. Calcd. for $\mathrm{C}_{13} \mathrm{H}_{8} \mathrm{~N}_{2} \mathrm{O}_{2} \mathrm{~S}$ (256.1): C, 60.9; H, 3.1; N, 10.9; S, 12.5\% Found: C, 60.9; H, 3.2; N, 10.7; S, 12.6\% IR (KBr, cm $\left.{ }^{-1}\right): 3149(\mathrm{NH}), 1657(\mathrm{CO}), 1628(\mathrm{CO}) ;{ }^{1} \mathrm{H}$ NMR (400 MHz, DMSO-d 6 ): $\delta$, ppm = 7.59-7.89 (m, 6H, Ar-H, CH-thiophene), 8.32 (s, 1H, CH-Pyrimidine), 12.85 (br, 1H, $\mathrm{NH}) \mathrm{D}_{2} \mathrm{O}$ exchangeable; ${ }^{13} \mathrm{C}$ NMR $\left(100 \mathrm{MHz}, \mathrm{DMSO}-\mathrm{d}_{6}\right): \delta, \mathrm{ppm}=126.1,129.3,129.5,130.6$, 133.7, 137.1, 139.0, 149.5, 158.3, 169.0, 188.6. MS: $m / z(\%) 256\left(\mathrm{M}^{+}, 100\right), 229$ (40), $179(80)$, 105 (100), 77 (80).

Ethyl 2-acetamido-5-benzoylthiophene-3-carboxylate (13b). Yield 45\%; mp. 133-35 ${ }^{\circ} \mathrm{C}$. Anal. Calcd. for $\mathrm{C}_{16} \mathrm{H}_{15} \mathrm{NO}_{4} \mathrm{~S}$ (317.0): C, 60.5; H, 4.7; N, 4.4; S, 10.1. Found: C, 60.6; H, 4.8; N, 4.6; $\mathrm{S}, 10.2$. IR (KBr, cm $\left.{ }^{-1}\right): 3264(\mathrm{NH}), 1674(\mathrm{CO}), 1633(\mathrm{CO}) ;{ }^{1} \mathrm{H}$ NMR (400 MHz, DMSO-d 6 ): $\delta$, $\mathrm{ppm}=1.23\left(\mathrm{t}, 3 \mathrm{H}, \mathrm{CH}_{3}, J=8 \mathrm{~Hz}\right), 2.36\left(\mathrm{~s}, 3 \mathrm{H}, \mathrm{CH}_{3}\right), 4.19\left(\mathrm{q}, 2 \mathrm{H}, \mathrm{CH}_{2}, J=8 \mathrm{~Hz}\right), 7.41-7.80(\mathrm{~m}$, $6 \mathrm{H}, \mathrm{Ar}-\mathrm{H}, \mathrm{CH}$-thiophene), 11.13 (br, 1H, NH) $\mathrm{D}_{2} \mathrm{O}$ exchangeable; ${ }^{13} \mathrm{C}$ NMR (100 MHz, DMSO$\left.\mathrm{d}_{6}\right): \delta, \mathrm{ppm}=15.1,61.8,107.3,114.3,123.5,129.3,129.6,132.4,133.5,138.1,154.4,164.7$, 169.8, 186.8. MS: $m / z(\%) 317\left(\mathrm{M}^{+}, 30\right), 275$ (100), 299 (85), 152 (25), 105 (80), 77 (55).

\section{Acknowledgements}

The authors are grateful to Kuwait University Research Administration for financial support of project SC11/02 and for SAF facilities project GS01/02 and GS03/01.

\section{References}

1. Gewald, K.; Schink, E.; Böttcher, H. Z. Chem. Ber. 1966, 99, 94.

2. Gewald, K.; Neumann, G.; Böttcher, H. Z. Chem. Ber. 1966, 6, 261.

3. Gewald, K.; Schink, E. Chem. Ber. 1966, 99, 2712.

4. Li, J. J. Name Reactions In Hetero Cyclic Chemistry, Wiley: New York, 2004; pp 193.

5. Peters, A. T.; Freeman, H. S. Advances In Color Chemistry Series "Modern Colorants Synthesis And Structure" Blackie Academic \& Professional, 1995; Vol. 3, pp 164.

6. Heaton, A. The Chemical Industry $2^{\text {nd }}$ Edn.; Blackie Academic \& Professional, 1994; pp 166.

7. Gregory, P. Chemistry And Technology of Printing And Imaging Systems Blackie Academic \& Professional, 1996; pp 260.

8. Barnes, D. B.; Haight, A. R.; Hameury, T.; Mclaughlin, M. A.; Mei, J.; Jedrow, J. S.; Toma, D. R. Tetrahedron 2006, 62, 11311.

9. Sabnis, R. W.; Rangnekar, D. W.; Sonawane, N. D. J. Heterocycl. Chem. 1999, 36, 333 and references therein.

10. Elnagdi, M. H.; Erian, A. W. Bull. Chem. Soc. Jpn. 1990, 63, 1854.

11. Elnagdi, M. H.; Negm, A. M.; Erian, A. W. Liebigs Ann. Chem. 1989, 1255. 
12. Al-Saleh, B.; Abdel-Khalik, M. M.; EL-Apasery, M. A.; Elnagdi, M. H. J. Chem. Res. 2005, 23.

13. Erian, A. W.; Hafez, E. A. A.; Darwish, E. S.; Elnagdi, M. H. Can. J. Chem. 1998, 76, 1038.

14. Abdel-Khalik, M. M.; Elnagdi, M. H. Synth. Commun. 2002, 32, 159.

15. Al-Saleh, B.; Abdel-Khalik, M. M.; Al Toukhy, A. M.; Elnagdi, M. H. J. Heterocycl. Chem. 2002, 39, 1035.

16. Abdel-Khalik, M. M.; Agamy, S. M.; Elnagdi, M. H. Synthesis 2001, 1861.

17. Al-Mousawi, S. M.; George, K. S.; Elnagdi, M. H. Diepharmazie 1999, 54, 571.

18. Abdel-Khalik, M. M.; Elnagdi, M. H.; Agamy, S. M. Synthesis 2000, 8, 1166.

19. Enezi, A.; Al-Saleh, B.; Elnagdi, M. H. J. Chem. Res. (S) 1997, 4; Ibid. (M), 110.

20. Al-Omran, F.; Al-Awadi, N.; Abdel-Khalik, M. M.; Kaul, K.; Elkhair, A. A.; Elnagdi, M. H. J. Chem. Res .(S) 1997, 84; Ibid. (M). 601.

21. Bacon, E. R.; Daum, S. J. J. Heterocycl. Chem. 1991, 28, 1953.

22. Elnagdi, M. H.; Abdaila, S. O. J. Prakt. Chem. 1973, 315, 1009.

23. Olomouc, C. R. J. Med. Chem., 2006, 49, 9500.

24. Audousset, M-P. U.S. Patent 6391063 BI, 2002: Chem. Abstr. 2002, 136, 294 A. 